\section{El análisis estratigráfico constructivo aplicado en casos específicos: los Baños Árabes de Hernando de Zafra (Granada) y la Galería Superior del Patio de los Arrayanes de la Alhambra de Granada}

\author{
Camilla Mileto, Fernando Vegas \\ Universidad Politécnica de Valencia
}

\begin{abstract}
Resumen
En el texto se presentan dos casos, muy diversos entre ellos, de aplicación del análisis estratigráfico constructivo. En el primer caso, el estudio de los Baños Árabes de Hernando de Zafra, el estudio estratigráfico constituía parte de un estudio previo más amplio y de un proyecto de restauración y recuperación de los espacios del baño, que podía proponer la demolición de una serie de elementos añadidos más recientes en el tiempo. El estudio estratigráfico tenía dos objetivos: por una parte la documentación de todos los elementos que se eliminarían posteriormente con la obra de restauración; por otra parte, la facilitación de la lectura de las diferentes conformaciones que el baño había tenido en el transcurso de su historia. En el segundo caso, el estudio de la Galería Superior del Patio de los Arrayanes de la Alhambra, el análisis estratigráfico constructivo constituía parte de un estudio más amplio, el estudio histórico constructivo. Este estudio fue desarrollado por un equipo formado por un historiador, un arquitecto técnico, dos arquitectos y un geólogo y se presenta como ejemplo de un estudio pluridisciplinar donde el trabajo se desarrolló realmente en equipo y no fue solamente el resultado de la adición apilada de diferentes trabajos.
\end{abstract}

Palabras clave: estratigrafía, documentación, proyecto, pluridisciplinar

\begin{abstract}
This text presents two quite distinct cases of the application of stratigraphic wall analysis. In the first case, the study of the Arab Baths at Hernando de Zafra, the stratigraphic study was part of a previous, wider study and project to restore and recover the baths, with the possibility of proposing the demolition of a series of more recently added elements. The stratigraphic study had two objectives: firstly, to document all elements to be eliminated later with the restoration work; and secondly, to facilitate the reading of the different formations that the baths had had throughout their existence. In the second case, the study of the Galería Superior del Patio de los Arrayanes de la Alhambra, the stratigraphic wall analysis was part of a wider study, the historic construction study. This study was developed by a team consisting of an historian, a technical architect, two architects and a geologist, and is presented as an example of a pluridisciplinary study where work was genuinely done in a team and was not simply the result of the combined sum of different studies.
\end{abstract}

Key words: stratigraphy, documentation, project, pluridisciplinary

\section{UN ESTUDIO ESTRATIGRÁFICO COMO DOCUMENTACIÓN DE FÁBRICAS DURANTE UNA OBRA DE RESTAURACIÓN: LOS BAÑOS ÁRABES DE HERNANDO DE ZAFRA (GRANADA) ${ }^{1}$}

El estudio estratigráfico constructivo de los Baños Árabes de Hernando de Zafra, conocidos como la Casa de la Tumbas, fue redactado por encargo la Junta de Andalucía, bajo sugerencia del proyectista, como parte de un estudio previo al proyecto de restauración más amplio.

El conjunto de los Baños ha sufrido una serie de intervenciones de reconversión de sus espacios interiores que han llevado a la fragmentación de las salas en un conjunto de pequeñas habitaciones de viviendas. La idea de la que partía el proyecto era la recuperación, al menos espacial, de las salas interiores del baño, idea que implicaba la demolición de las actuales particiones interiores.

Considerando la posibilidad de que el proyecto contemplara la remoción de los tabiques y de las construcciones recientes, el estudio se propuso dos objetivos principales. Por un lado, proporcionar una documentación de todos los elementos, para que quede constancia de los mismos, una vez eliminados. Por otro lado, ofrecer una visión general de los elementos en relación con su época de construcción, que facilite tanto una lectura inmediata de los elementos recientes superpuestos a los periodos precedentes, como una idea de las partes de los Baños ocultas por estos mismos elementos recientes y que, en consecuencia, podrían recuperarse con la remoción de los estratos de menor antigüedad.

\subsection{Los Baños Árabes de Hernando de Zafra en Granada}

El conjunto conocido como Baños Árabes de Hernando de Zafra o Casa de las Tumbas, se encuentra en el lado meridional de la Calle Elvira, o sea la antigua calle principal de la medina de Granada. Actualmente el acceso se crea desde la calle de San Andrés que toma prestado su nombre de la iglesia que fue construida en el lugar de una antigua mezquita, quizás relacionada con el baño. Actualmente el baño se encuentra encajonado en el interior de una manzana de

\footnotetext{
${ }^{1}$ El análisis estratigráfico constructivo de los Baños de Hernando de Zafra en Granada ha sido encargado por la Junta de Andalucía, en el año 2001, a los arquitectos Camilla Mileto y Fernando Vegas. Se trata de un estudio realizado en el ámbito del Estudio Previo de los Baños que tiene que preceder el Proyecto Básico de Rehabilitación del edificio, asignado al arquitecto Antonio Luis Espinar Moreno por la misma Junta de Andalucía. Han colaborado a la realización del trabajo: Bruno Rives Calbet (arqueólogo); Raquel Martínez Padilla e Inmaculada Oliver Monleón (estudiantes de último curso de la ETSA de la Universidad Politécnica de Valencia)
} 
edificios más recientes, posiblemente construidos entre el XVIII y el XIX, tanto que el conjunto no se puede apreciar externamente porque permanece completamente tapado por los edificios que le rodean.

La construcción del baño se remonta posiblemente a un periodo comprendido entre el siglo XII y el siglo XIII y, probablemente, se mantuvo en uso hasta el siglo XV. Se componía de una sala vestidor (apoditherium) una sala fría (frigidarium), de una sala templada (tepidarium) y de una sala caliente (caldarium), además de la caldera y del horno. Las salas estaban cubiertas por bóvedas esquifadas con lucernas, algunas de las cuales se conservan hoy en día de manera integral, mientras que otras se adivinan a través de las huellas.

Posiblemente el baño se reconvirtió a otra función entre el siglo XVI y el XVIII y, finalmente, fue destinado a viviendas en los siglos XIX y XX. Actualmente, de hecho, el cuerpo de los Baños se presenta como un conjunto de estancias interiores definidas, en su conformación actual, posiblemente entre el siglo XIX y principios del siglo XX, cuando, los amplios espacios del baño se redujeron en pequeñas habitaciones para su ocupación como viviendas. Con esta finalidad, se subdividieron los espacios iniciales con una serie de tabiques y paredes que impiden la percepción de los espacios iniciales.

\subsection{Descripción de la hipótesis de los periodos constructivos de los Baños de Hernando de Zafra (Fig. 1 y 2)}

En las fábricas estudiadas, no se disponía, en el momento de la redacción del estudio, del respaldo de una investigación histórica para comparar la cronología relativa obtenible del análisis estratigráfico con una cronología absoluta. A falta de una documentación histórica exhaustiva se pudo plasmar una hipótesis de periodos constructivos, sustancialmente en base a una cronología relativa, identificando tres periodos principales, divididos a su vez en una serie de fases. Sin embargo, se intentó relacionar estos periodos con algunos periodos históricos con el objetivo de poder plasmar, de todos modos y aunque sea aproximada, una hipótesis de las fases constructivas de los baños. Las fechas se han supuesto sobre la base de un análisis de los elementos cronotipológicos identificados en los baños: tipos de fábricas murarias, tipos de enlucidos, tipos de huecos, etc.

En consecuencia, los periodos propuestos son: el primero, desde la construcción del baño (posiblemente entre el siglo XII y el siglo XIII) hasta probablemente el siglo XV; el segundo, desde el siglo XVI en adelante, probablemente hasta el siglo XVIII; y el tercero que consiste en los siglos XIX y XX.

\section{Periodo 1}

El primer periodo se refiere supuestamente a la fase que va desde la construcción del baño, posiblemente entre el siglo XII y el siglo XIII, hasta posiblemente finales del siglo XV cuando, al prohibirse el uso de los baños, posiblemente el conjunto perdió su función. Corresponde en la actualidad a una serie de restos visibles y a partes actualmente no visibles porque están escondidas por añadidos sucesivos. Corresponden a este periodo: una serie de fábricas de tapial calicostrado, en las que se conserva en gran parte la costra; unas fábricas de ladrillo, correspondientes tanto a las partes altas de los muros y a las bóvedas de las partes laterales de la sala templada, como a las estructuras de los aljibes con restos de enlucidos, como a los restos del arco del pasaje entre la sala templada y la sala meridional, como a los arcos que subdividían la sala templada, con sus columnas y capiteles de mármol. En este mismo periodo se han considerado los muros verticales aparecidos tras la excavación arqueológica.

\section{Periodo 2}

El segundo periodo corresponde posiblemente al momento en que los baños, perdida su función inicial, sufren las primeras transformaciones. Se considera la posibilidad de un periodo flexible, posiblemente identificable con el siglo XVI que, sin embargo, podría alargarse hasta el siglo XVIII. Se considera perteneciente a este arco temporal, sobre todo, la estructura del muro más meridional del baño, construida con machones de ladrillo y cajones a su vez de ladrillo, con un nivel ligeramente rehundido respecto a la superficie de los machones y revestidos con un enlucido de cal. También, se debe subrayar que este muro se construyó inclinado respeto a la dirección del baño, posiblemente vinculado a otras construcciones y destinado a posibilitar una circulación alrededor del mismo baño. Su realización con una cierta inclinación obligó al picado de la pared a la cual se enfrentaba de manera no paralela, para disminuir su espesor y forzar a las mismas a un cierto paralelismo. El picado del muro se puede leer muy claramente en algunas partes del mismo. El segundo periodo comprende además una serie de pequeñas modificaciones de dos huecos presentes en estas mismas paredes.

\section{Periodo 3}

El tercer periodo constructivo corresponde a un periodo de tiempo que se extiende desde las intervenciones del siglo XIX hasta nuestros días y se divide a su vez en tres fases. La primera corresponde a las intervenciones realizadas en el siglo XIX cuando se convierte el baño en viviendas y sus espacios se subdividen en pequeñas habitaciones. Se trata, so- 
3.

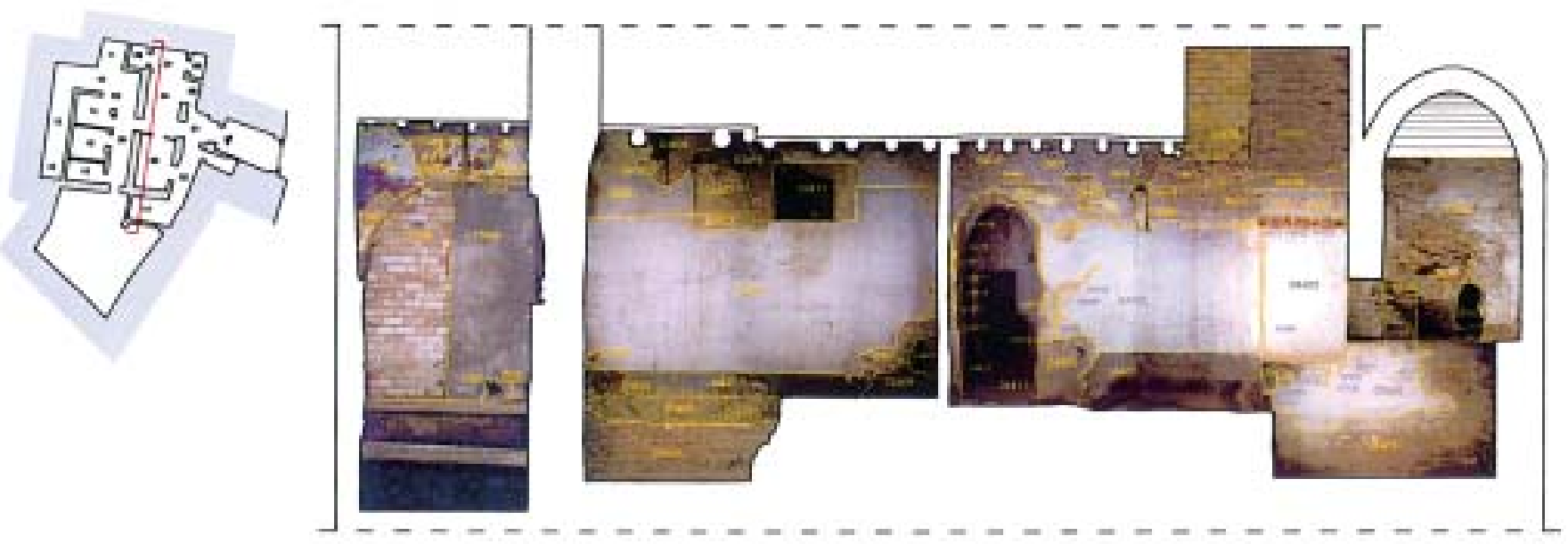

Fig. 1. Plano de análisis estratigráfico. Sección longitudinal de los Baños Árabes de Hernando de Zafra (Granada)

5

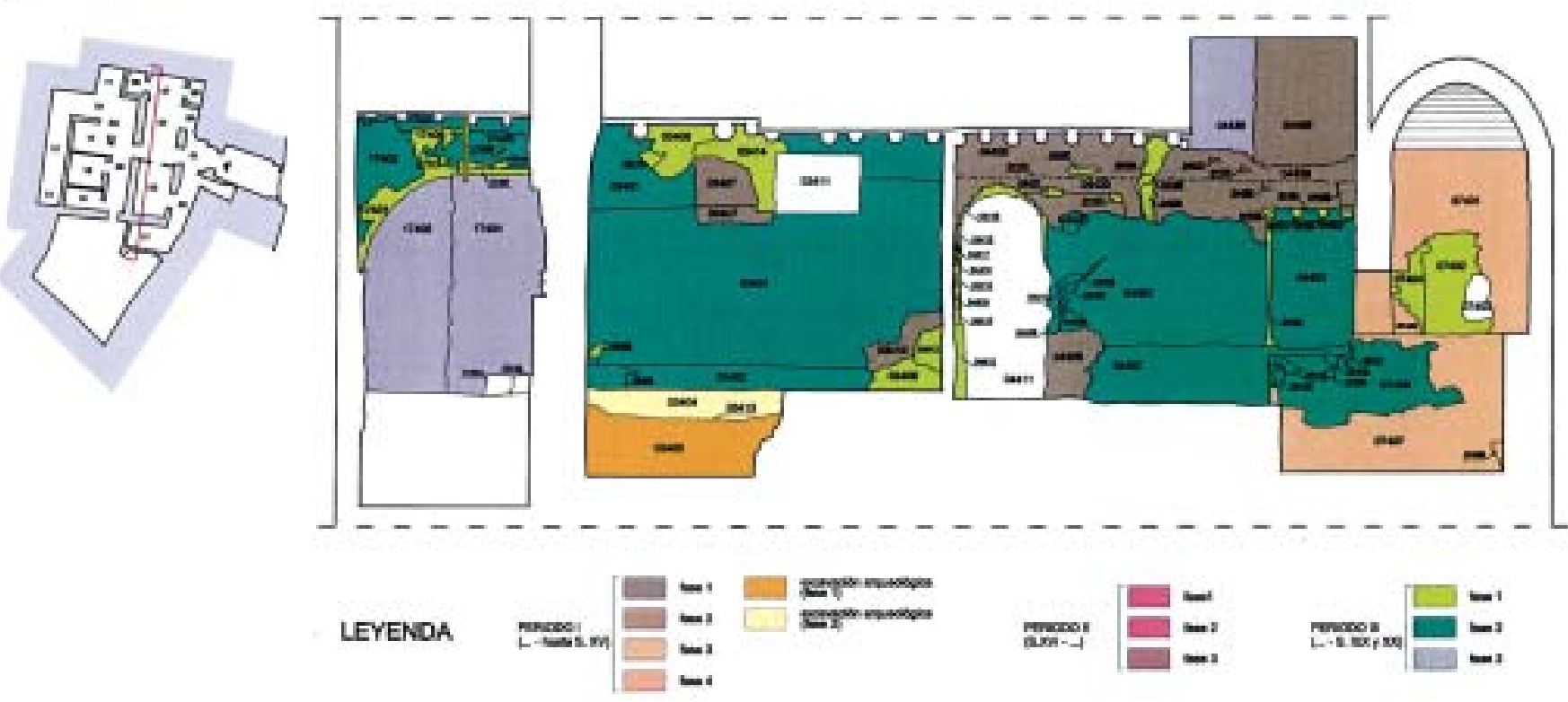

Fig. 2. Plano de hipótesis de periodos constructivos. Sección longitudinal de los Baños Árabes de Hernando de Zafra (Granada) 
bre todo, de una serie de tabiques de pequeño espesor, construidos de ladrillo, que fraccionan los grandes espacios $\mathrm{y}$, con este mismo objetivo, llegan a tabicar los arcos de herradura de la sala templada, envolviendo sus columnas. Con el mismo fin de adecuación del baño a viviendas se abrieron una serie de huecos en los muros perimetrales. En esta misma fase se realizó la demolición de las grandes bóvedas de la sala templada y de la sala meridional, para poder añadir un segundo piso al conjunto. La segunda corresponde, principalmente, a una serie de enlucidos realizados en el tiempo sobre las mismas paredes de la fase sucesiva, pero en ella no destaca ninguna intervención relevante. La tercera fase corresponde a las obras realizadas durante el año 2000-2001 para proteger el conjunto de los agentes atmosféricos (clausura de huecos y colocación de una cubierta de uralita).

\subsection{La realización de un análisis estratigráfico constructivo durante una obra de restauración arquitectónica}

El estudio estratigráfico constructivo se realizó fundamentalmente en las estancias interiores del conjunto, analizando las fábricas correspondientes a todos los periodos tanto constructivos como derivados de transformaciones posteriores. El estudio debía proporcionar una documentación lo más exhaustiva posible con el doble objetivo, propuesto por el proyectista, por una parte, de documentar los elementos que podían desaparecer con las demoliciones decididas por el proyecto y, por otra parte, de facilitar la lectura de las partes añadidas respecto a las originales.

Si por un lado, se trataba de proporcionar un verdadero registro arqueológico, o sea una documentación completa del estrato visible que pudiera servir, una vez que este último se elimine para leer el estrato inferior, como modelo de lo demolido, o sea como fuente primaria de información en lugar de la fuente física demolida. Por otra parte, se trataba de identificar los elementos que habían sido añadidos en los últimos tiempos para que pudieran ser objeto de demolición y recuperar el estrato inferior, más antiguo.

No se pretende, en este texto, entrar en el juicio de la validez de las decisiones de proyecto, simplemente se considera importante destacar la labor que el método de análisis estratigráfico puede realizar al servicio del proyecto de restauración, tanto si adopta una postura muy conservadora como una más transformadora.

Si se piensa que la demolición de los añadidos se realizará demoliendo casi completamente las unidades estratigráficas pertenecientes a una misma fase constructiva (la más reciente), se puede comparar la demolición a una excavación arqueológica, donde se elimina cada vez completamente un estrato. En el caso de la excavación arqueológica se vuelve a repetir el registro de las unidades, cada vez que se baje un estrato de manera de obtener el registro completo de todos los estratos.

De acuerdo con estas reflexiones, se considera de gran importancia la posibilidad de realizar una segunda etapa del estudio de análisis estratigráfico constructivo, una vez concluidos los trabajos de excavación arqueológica y demolición de las partes recientes. El objetivo principal de esta segunda fase será, por una parte, la compleción de la documentación con todos los elementos anteriormente ocultos y, por otra parte, la formalización de una nueva hipótesis de fases constructivas, a la luz de los nuevos datos aparecidos.

De gran interés sería, además, la posibilidad de realizar una tercera fase de análisis, ya no ligada a la obra en curso, sino a los resultados de la misma. Se podría realizar una fase de registro una vez terminada la obra con el objetivo de evaluar la entidad e importancia de los datos eliminados o añadidos. Se trataría de un análisis estratigráfico al servicio de la reflexión sobre el proyecto y su impacto sobre lo construido.

\section{UN TRABAJO INTERDISCIPLINAR: LA GALERÍA SUPERIOR DEL PATIO DE LOS ARRAYANES DE LA ALHAMBRA DE GRANADA}

El estudio de la sala de la Galería Superior del Patio de los Arrayanes se desarrolló en el ámbito de un estudio históricoconstructivo más amplio, encargado por el Patronato de la Alhambra y el Generalife, con el objetivo de adquirir un mayor conocimiento de un sector de la Alhambra que, a pesar de su reducido tamaño, recoge un buen número de intervenciones en el transcurso del tiempo. El estudio fue encargado a un equipo formado por diferentes profesionales (Miguel Sorroche, historiador, José Manuel López Osorio, arquitecto técnico, Camilla Mileto y Fernando Vegas, arquitectos, Francisco Martín Peinado, geólogo), donde cada uno debía desarrollar una parte concreta del mismo en estricta relación con las demás partes: un estudio histórico documental, un estudio de las técnicas constructivas, un análisis estratigráfico constructivo y un estudio de caracterización de los materiales.

El estudio se realizó en las paredes interiores de la sala central de la Galería Alta de la Nave de Poniente del Patio de los Arrayanes y en las paredes de la pequeña sala de conexión entre dicha Galería y el Palacio de Carlos V. Además, se ha extendido el estudio a la fachada exterior oeste de la Nave de Poniente. 


\subsection{La Galería Alta de la Nave de Poniente del Patio de los Arrayanes}

La fábrica estudiada se identifica como el espacio de la planta superior de la crujía occidental del Patio de los Arrayanes, que se extiende desde la Sala de la Barca a norte, hasta el Palacio de Carlos V a sur y que, abierto hacia el Patio de los Arrayanes, vuelve la espalda al Mexuar. El ámbito del estudio se caracteriza por ser una zona en la que confluyen tres diferentes áreas, cada una de gran importancia y relevancia histórica: por un lado, el Palacio de Carlos V, cuya construcción, empezada en 1535, afectó la parte meridional de la galería, en busca de una nueva conexión entre el nuevo Palacio y la Casa Real Vieja (palacios nazaríes); por otro lado, el Mexuar, de cuyas primitivas dependencias permanecían las huellas en el exterior de la pared occidental de la galería, cerca de la entrada a los palacios; por último, el mismo Palacio de Comares, al que pertenece la galería estudiada.

Sin duda, se trata de una de las zonas más atractivas de todo el conjunto, ya que en ella se centra el problema irresuelto de las intervenciones realizadas para la construcción del palacio renacentista.

\subsection{La metodología adoptada (Fig. 3)}

La fábrica considerada, la Galería Alta de la Nave de Poniente del Patio de los Arrayanes, se presentaba, en el momento de la realización del estudio, legible por lo que atañe a los paramentos interiores de la sala central y de la pequeña estancia de conexión entre dicha galería y el Palacio de Carlos V. En ese momento, no era, sin embargo, legible la fachada de dicho ala hacia el Patio de los Arrayanes, que estaba enlucida, ni tampoco resultaba legible la fachada occidental que se acababa de enfoscar.

Por ello, el estudio se centra sobre todo en los paramentos interiores de la Galería Alta, sin olvidar, sin embargo, la importancia de una lectura conjunta de las dos caras del muro. Por esta razón, se ha intentado recuperar, al menos en parte, la información relativa a la fachada oeste, legible hasta hace poco mediante el montaje de fotografías de archivo de la fachada antes de la intervención.

\subsection{Una reflexión sobre el tema del trabajo interdisciplinar}

El caso del estudio de la Galería Alta se presentó, no sólo por el interés del edificio estudiado, sino sobre todo en el óptica de reflexionar sobre el valor insustituible de un trabajo desarrollado de manera interdisciplinar. Trabajo interdisciplinar, en el caso específico, no significa simplemente que varios especialistas estén trabajando, desde diferentes puntos de vista, sobre el mismo objeto, sino más bien que el trabajo se ha desarrollado, aunque sea en documentos separados, por parte de un equipo en el cual el intercambio de información y el recíproco asesoramiento ha sido el presupuesto fundamental. No se ha tratado, de hecho, de reco-
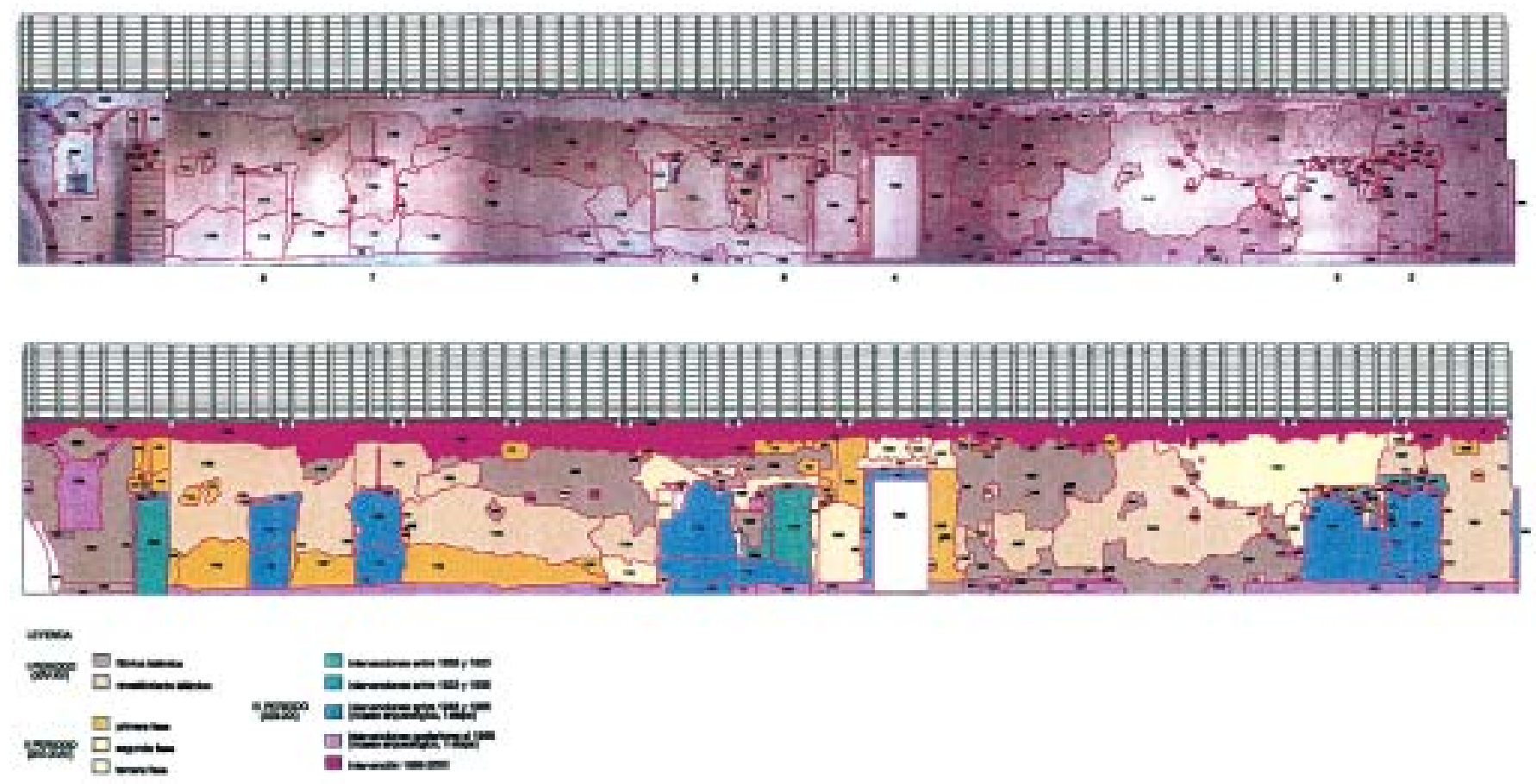

Fig. 3. Plano de análisis estratigráfico y plano de hipótesis de períodos constructivos del alzado Oeste interior de la Galería Alta de la nave de Poniente del Patio de los Arrayanes en la Alhambra (Granada) 
ger al final los diferentes trabajos para darle una forma común, sino de trabajar conjuntamente desde el primer día.

Sin duda, el objeto de estudio se prestaba especialmente a este tipo de coordinación, dada la cantidad de información que se podía recoger en todos los ámbitos estudiados, desde la documentación histórica e iconográfica, a la documentación sobre las intervenciones de restauración, el estudio de la estratigrafía constructiva, el estudio de las técnicas constructivas, hasta el análisis de los materiales. Sin embargo, desde principio se ha trabajado con el objetivo de que todos los datos, al final, pudiesen concluir en un único trabajo, cuya única aspiración era el ampliar, con un estudio riguroso, los conocimientos sobre esta parte del conjunto monumental.

En ningún momento, se ha deseado forzar las interpretaciones de los datos para alcanzar posibles soluciones $\mathrm{y}$, de hecho, se ha pretendido simplemente recoger una serie de datos que, más que respuestas, abren una serie de opciones y líneas de interpretación. En consecuencia, donde no se podía encontrar una respuesta cierta, se ha preferido responder con interrogantes y cuestiones abiertas. 